\title{
Cytological Aspects of Equine Oral Fibrosarcoma
}

\author{
Danieli Brolo Martins', Cristina Krauspenhar Rossato ${ }^{2}$, Nadjanaira Barbosa Abrão ${ }^{1}$ \& Mariela da Luz²
}

\begin{abstract}
Background: Although mesenchymal neoplasms are prominent in horses due to the occurrence of sarcoids, other neoplasms may occur. Fibrosarcoma in horses is uncommon, notwithstanding, few studies have performed diagnostic techniques. It is presumed, therefore, that other diagnostic methodologies should be explored. In this sense, fine needle aspiration cytology (FNAC) emerges as an effective, low-cost, and minimally invasive method to identify tumors in these animals. The objective of this study was to describe the use of FNAC in the diagnosis of oral fibrosarcoma in horses. Once fibrosarcoma is an aggressive tumor, it is important to validate effective techniques for an early diagnosis.

Case: A 10-year-old male Quarter Horse was treated, with a clinical complaint of increased volume in the right maxillary region. This tumor occupied about $1 / 4$ of the hard palate area. Hyporexia, hypodipsia, mild dehydration, and weight loss were observed at clinical examination. Complementary examinations were requested, and an extensive radiolucent mass with an indication of bone involvement was observed on the radiograph. The hemogram revealed normocytic normochromic anemia, in addition to lymphopenia. In the serum biochemistry test, AST (aspartate aminotransferase) and creatinine were slightly increased. FNAC showed a malignant mesenchymal neoplasm, possibly fibrosarcoma. Due to its clinical worsening and lack of response to supportive treatment, humanitarian euthanasia was performed. Finally, a histopathological examination confirmed the oral fibrosarcoma suggested by FNAC.

Discussion: The early diagnosis of neoplasms is important for its correct treatment, as well as the prognosis. In this way, the combined use of complementary tests helps in the resolution of the case. FNAC is still little used in the examination routine in horses. In the present report, this test demonstrated efficacy since it revealed mesenchymal cells compatible with fibroblasts, in addition to malignancy characteristics such as cells with anisocytosis, cytoplasmic basophilia, macrocariosis in some cells, round to elongate nuclei, coarse chromatin, prominent nucleoli, and a discrete eosinophilic amorphous matrix between the cells. The other laboratory findings were consistent with the disease. Normocytic normochromic anemia is found in chronic conditions, such as neoplasms, and lymphopenia may be a finding in stress situations. The anatomical location of the tumor and the bone involvement made surgical treatment impossible. With this, we sought to alleviate the patient's discomfort with supportive therapy, which in situations of chronicity is not always effective, as in the case described. In conclusion, FNAC can be used to differentiate malignant tumors from those considered benign, as well as in the identification of tumor groups. Despite the high predictive value of FNAC, histopathological examination was fundamental for the categorization of the neoplasia. Therefore, it is recommended to confirm the FNAC result by histopathology since equine fibrosarcoma can be easily confused with other malignancies more common in the species, such as sarcoids. The cytological examination showed a correct orientation in this case. Nonetheless, further studies are needed to demonstrate the applicability of this technique in increased oral volume in horses.
\end{abstract}

Keywords: oral tumor, malignant mesenchymal neoplasm, fine needle aspiration cytology, horse. 


\section{INTRODUCTION}

Although mesenchymal neoplasms stand out in horses due to the significant participation of sarcoids [10], other cancers belonging to the same group of cells have lower appearance. [12] studied 315 biopsies of horses and found only one case of fibrosarcoma.

Fibrosarcoma is unusual in horses and difficult to be distinguished from other neoplams $[3,6]$. Moreover, it is clear that its diagnosis occurs most often through histopathological examination $[3,7,12,13]$. Just few studies have used auxiliary techniques for diagnosis [6,9], which allows to presume that other diagnostic methodologies should be explored.

In this aspect, fine needle aspiration cytology (FNAC) emerges as a cheap, fast, and minimally invasive method to identify the animal's tumor [14]. By this technique, it is possible to maximize the chances of a correct diagnosis, directing the clinician to a more appropriate medical management, whether in therapy or in prognosis.

Thus, this paper describes the use of FNAC for the diagnosis of oral fibrosarcoma in a horse. Once fibrosarcoma is an aggressive tumor [3], it is important to validate effective techniques for an early diagnosis.

\section{CASE}

A 10-year-old male Quarter horse, weighing $460 \mathrm{~kg}$ was attended. The horse presented swelling of the right maxillary region (Figure 1A) for the last 60

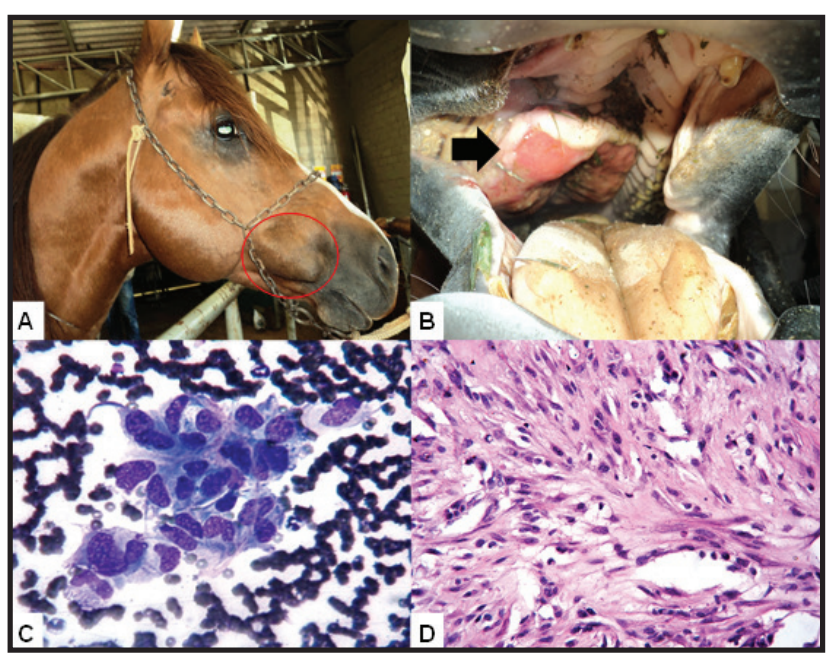

Figura 1. Equine with oral fibrosarcoma. A- Increase in volume next to the right side of the face (detail). B- Mass occupying the region of the hard palate (arrow). C- Cytological examination evidencing agglomeration of mesenchymal cells presenting characteristics of malignancy [Panótico, 1000x]. D- Histopathological examination evidencing neoplastic fibroblasts [HE, 40x]. days. Appetite loss, weight loss, and hypodipsia were also observed. On physical examination, the mass was localized around $1 / 4$ of the hard palate area (Figure 1B). Slight dehydration was verified. The other physiological parameters were normal.

Additional tests were requested. The radiographic images showed an extensive radiolucent mass indicating bone involvement. FNAC showed mesenchymal cells with anisocytosis, cytoplasmic basophily, macrocariosis in some cells, rounded to elongated nuclei, coarse chromatin, prominent nucleoli, and discrete eosinophilic amorphous matrix among the cells (Figure 1C). The cytological examination revealed malignant mesenchymal neoplasm, possibly oral fibrosarcoma.

Subsequently, histopathological examination was performed (Figure 1D), which confirmed FNAC results. Due to the extent of the mass that did not allow a surgical excision, supportive treatment was indicated to improve the quality of life of the animal. However, due to the gradual deterioration of the horse, the owner chose for humane euthanasia of the patient, three months after the diagnosis of the tumor.

\section{DISCUSSION}

Neoplastic processes are relevant causes for veterinary care in equids [1] and the use of a combination of diagnostic techniques can provide greater security in the final result [6]. Thus, the early diagnosis of the neoplasm is important for the correct treatment and prognosis.

The interpretation of cytological samples in veterinary medicine should be carried out with extensive knowledge, taking into account the different species of animals. The cytologist should know the frequency and biologic behavior of neoplastic lesions according to the analyzed species, as well as breed and handling conditions of the patient [14]. Although the FNAC is an examination in full expansion in veterinary routine services, this technique has not been much applied in equine species [8], being underutilized in some cases.

In fibrosarcoma cytology, the cells may present rounded, oval, or even star-like shape, and a single indistinct cytoplasm tail. Multinucleated fibroblasts can also be found. Slight to intense variation in the size, shape, nuclei and nucleoli of the cells is also observed, as well as other features like cytoplasm basophily, increase in nuclei:cytoplasm ratio, and angular nucleoli or macronuclei $[11,15]$. The cells may be associated with collagenous eosinophilic material [4], as seen in 
this case. The cytology sample of the patient released a moderate amount of individualized and grouped cells.

Interestingly, several of the cell characteristics presented for fibrosarcoma may develop as the malignancy potential increases [15]. In the cytological sample of the horse, similar cell characteristics to those described in the literature were observed, which suggests the malignancy process.

As demonstrated in this case, cytology can be used to differentiate malignant tumor processes from those considered benign. The sensitivity, specificity, and positive predictive value of FNAC for the diagnosis of soft tissue tumors may reach about $91.5 \%$, $92.5 \%$, and $95.5 \%$, respectively. However, this technique may be less effective in the exact categorization of tumors [2].

Furthermore [5], investigating oral tumors in dogs through exfoliative cytology and histopathology, found $100 \%$ agreement between both techniques to fibrosarcoma, which was considered a good result. Nevertheless, the authors considered a low number of sampling studied, since this is also a rare neoplasm in the oral cavity of dogs.

Thus, histopathologic examination was fundamental for a subsequent confirmation of the tumor in our patient, as sarcoid and other granulation tissue can easily be mistaken for fibrosarcoma when evaluated only by FNAC [15].

The imaging findings were consistent with the disease presented by the animal. Radiographic examination presented the local bone lysis and excluded the existence of advanced metastasis [5]. Thus, FNAC, in addition to the other complementary tests, enabled a better understanding of the evolution of the disease in the horse and assisted in its prognosis.

Our animal's nodule showed a large volume, was in a delicate area for surgical removal, and showed evidence of bone destruction in the maxillary region by the neoplasia. There was displacement of molar teeth and the presence of ulceration, which could have contributed to the discomfort and pain that the animal felt. The fibrosarcoma is poorly circumscribed and may ulcerate [4], with the potential to be locally invasive and destructive. Although the absence of recurrence has been reported [13], a local recurrence after surgical excision has been observed [3]. This implies the need for broad and defined margins [13], which is not always possible in the oral region, which makes it difficult or even prevents surgical treatment.

Therefore, euthanasia was performed on the animal due to its advanced critical state, which ended up jeopardizing the outcome, in addition to the restricted financial conditions of the owner. This method has also been recommended previously in similar situations [9].

The cytological examination presented a correct direction in this case. However, there are few studies that demonstrate the applicability of this technique in increased oral volume in horses. Once the equine fibrosarcoma can be easily mistaken with other most common malignant neoplasms in the species, such as sarcoid, confirming FNAC result by histopathology is recommended.

Declaration of interest. The authors report no conflicts of interest. The authors alone are responsible for the content and writing of the paper.

\section{REFERENCES}

1 Baccarin R.Y.A., Silva L.C.L.C., Belli C.B., Fernandes W.R. \& Zoppa A.L.V. 2011. Ocorrência de neoplasias em 15 anos de atendimento hospitalar de equídeos. Brazilian Journal of Veterinary Research and Animal Science. 48 (6): 439-445.

2 Dey P., Mallik M.K., Gupta S.K. \& Vasishta R.K. 2004. Role in fine needle aspiration cytology in the diagnosis of soft tissue tumours and tumours-like lesions. Cytopathology. 15: 32-37.

3 Dixon P.M. \& Head K.W. 1999. Equine nasal and paranasal sinus tumours: part 2: a contribution of 28 case reports. Veterinary Journal. 157: 279-294.

4 Dunn J. 2014. Cytology of cutaneous and subcutaneous lesions. In: Manual of Diagnostic Cytology of the Dog and Cat. Devon: WileyBlackwell, pp.57-88.

5 Felizzola C.R., Stopiglia A.J. \& Araújo N.S. 1999. Oral tumors in dogs. Clinical aspects, exfoliative cytology and histopathology. Ciência Rural. 29: 499-506.

6 Findley J.A., Singer E.R., Milner P.I. \& Leeming G.H. 2014. Use of immunohistochemical staining and electron microscopy to aid in diagnosis of soft tissue sarcomas associated with the fetlock joint in two horses. Journal of Veterinary Diagnostic Investigation. 26(3): 465-469. 
7 Kannegieter N.J., Schaaf K.L., Lovel D.K., Simon C.D. \& Stone B.M. 2010. Myofibroblastic fibrosarcoma with multifocal osseous metaplasia at the site of equine influenza vaccination. Australian Veterinary Journal. 88(4): 132-136.

8 Ortiz L.C., Garzón A. \& Rodríguez B.J. 2011. Tendencias citodiagnósticas en el Laboratorio de Patología Animal de la Universidad de Antioquia (Colombia). Revista Colombiana de Ciencias Pecuarias. 24: 157-169.

9 Pezzanite L.M., Devine D.V. \& Toll L. 2015. Soft tissue sarcomas in the pharyngeal region of a 5-year-old Quarter Horse mare. Equine Veterinary Education. 28(12): 661:664.

10 Ramos A.T., Souza A.B., Norte D.M., Ferreira J.L.M. \& Fernandes C.G. 2008. Tumores em animais de produção. Ciência Rural. 38(1): 148-154.

11 Raskin R.E. 2011. Pele e tecido subcutâneo. In: Raskin R.E. \& Meyer D.J. (Eds). Citologia Clínica de Cães e Gatos. 2.ed. Rio de Janeiro: Elsevier, pp.26-76.

12 Sharkey L.C. \& Seelig D.M. 2014. All Lesions Great and Small, Part 1: Diagnostic Cytology in Veterinary Medicine. Diagnostic Cytopathology. 42(6): 535-543.

13 Souza T.M., Brum J.S., Fighera R.A., Brass K.E. \& Barros C.S.L. 2011. Prevalência dos tumores cutâneos de equinos diagnosticados no Laboratório de Patologia Veterinária da Universidade Federal de Santa Maria, Rio Grande do Sul. Pesquisa Veterinária Brasileira. 31(5): 379-382.

14 Story M.R., Gaughan E.M., Andrews G.A. \& Balch S. 2005. Fibrosarcoma over the tarsal groove of a 14-month-old Quarter horse. Veterinary and Comparative Orthopaedics and Traumatology. 18(2): 115-118.

15 Tyler R.D., Meinkoth J.H., Cowell R.L., MacAllister C.G. \& Caruso K.L. 2002. Cutaneous and subcutaneous lesions - masses, cysts and fistulous tracts. In: Cowell R.L. \& Tyler R.D. (Eds). Diagnostic Cytology and Hematology of the Horse. 2nd edn. Mosby: New York: Mosby, pp.19-42. 